\title{
DIGITAL PRESERVATION OF THE QUON SANG LUNG LAUNDRY BUILDING, FORT MACLEOD, ALBERTA.
}

\author{
Peter Dawson $^{1}$, Farzan Baradaran ${ }^{4}$, Adam Jahraus ${ }^{5}$, Elisa Rubalcava ${ }^{2}$, Alireza Farrokhi ${ }^{3}$, Christina Robinson ${ }^{6}$. \\ ${ }^{1}$ Professor, Department of Anthropology and Archaeology, University of Calgary \\ ${ }^{2}$ Heritage Conservation Technologist, Alberta Culture and Tourism: Historic Resources Management Branch \\ ${ }^{3} \mathrm{Head}$ of Conservation and Construction Services, Alberta Culture and Tourism: Historic Resources Management Branch \\ ${ }^{4} \mathrm{PhD}$ Student, Faculty of Environmental Design, University of Calgary \\ ${ }^{5}$ Technician, Department of Geomatics Engineering, University of Calgary. \\ ${ }^{6} \mathrm{PhD}$ Student, Department of Anthropology and Archaeology, University of Calgary.
}

KEY WORDS: Preservation, Emergency recording, Digital modeling, Laser scanning.

\begin{abstract}
:
This paper describes the results of an emergency recording and archiving of a historic structure in Southern Alberta and explores the lessons learned. Digital recording of the Quon Sang Lung Laundry building in Fort Macleod, Alberta, was a joint initiative between Alberta Culture and Tourism and the University of Calgary. The Quon Sang Lung Laundry was a boomtown-style wood structure situated in the Fort Macleod Provincial Historic Area, Alberta. Built in the mid-1800s, the structure was one of the four buildings comprising Fort Macleod's Chinatown. Its association with Chinese immigration, settlement, and emergence of Chinese-owned businesses in early twentieth-century Alberta, made the Quon Sang Lung Laundry a unique and very significant historic resource. In recent years, a condition assessment of the structure indicated that the building was not safe and that the extent of the instability could lead to a sudden collapse. In response, Alberta Culture and Tourism engaged the Departments of Anthropology and Archaeology and Geomatics Engineering from the University of Calgary, to digitally preserve the laundry building. A complete survey including the laser scanning of all the remaining elements of the original structure, was undertaken. Through digital modeling, the work guarantees that a three-dimensional representation of the building is available for future use. This includes accurate 3D renders of the exterior and interior spaces and a collection of architectural drawings comprising floor plans, sections, and elevations.
\end{abstract}

\section{INTRODUCTION}

The history of Chinese immigration, especially in western Canada, is synonymous with the many hand laundry shops that operated in towns and cities across the country. The construction of the Canadian Pacific Railway (1880-1885) and the Caribou Wagon Road (1863-64), made western Canada one of the most appealing places for Chinese immigrants during the 19th century (Hoe, 2003; Marshall, 2009). When these projects were completed, many Chinese sought out new economic opportunities. Despite the scarcity of labor in Canada, numerous émigrés faced racism which resulted in social and economic segregation from mainstream society (Hoe, 2003). The operation of laundry shops became a means by which many Chinese laborers could earn a living, albeit a meager one (Hoe, 2003; Wang., 2002). However, the hours were long, and the work of hand washing laundry was monotonous and painful. The laundry shops themselves were often viewed with suspicion by outsiders, who saw them as unclean, and as dens for opium smoking and gambling by the Chinese bachelors living within them (Hoe, 2003, Morrison et al., 2013). By the 1950's many of these laundry shops had been replaced by White-owned Industrial Steam Laundries, as well as the rise of home washing machines (Hoe, 2003; Wang, 2002). A few of these nowabandoned Chinese laundry shops remain in small towns throughout Canada. They are important heritage sites because of their relationship to Chinese immigrant history. However, many are at risk of being lost to development, or are facing demolition because the buildings are hazards.

In this paper, we discuss how terrestrial laser scanning and aerial photogrammetry were used to digitally preserve the Quan Sang Lung Laundry Shop in Fort Macleod, Alberta. Developing a workflow to digitally capture and archive iconic heritage sites is one objective. But another equally important objective is to use the resulting digital models to communicate the experiences of Chinese immigrants. With the recent rise in anti-immigrant sentiments in North America and Europe, there has arguably never been a greater need to keep the histories and experiences of earlier immigrant periods alive in the collective memories of mainstream Canadian society.

\section{PROJECT CONTEXT}

\subsection{Historical Background}

Built in 1907, the Quon Sang Lung Laundry was evidence of the emergence of a sizeable Chinese population in early twentieth-century Alberta. While there were few Chinese in Alberta at the turn of the last century, the province's booming economy attracted thousands of Chinese immigrants. Chinese merchants opened small businesses, such as laundries, restaurants and corner groceries, across the province. Chinese laundries became a characteristic feature of early twentiethcentury Albertan towns and cities, as Chinese merchants filled a niche in labour-intensive retail businesses left open by other entrepreneurs. The Quon Sang Lung Laundry was part of a larger commercial network in Fort Macleod from 1907 through 1919; owned by a successful Chinese retailer, Quon Sang Lung and his brother. The structure reflected the history of Chinese immigration, settlement, adaptation and contribution to Alberta's growth in the early twentieth century.

\subsection{Description of the site}

The Chinese Laundry is located in an area which was once the core of Fort Macleod's Chinatown. Three or four buildings on the corner of 25th Street and 3rd Avenue comprised the main Chinatown district. Prior to 1900 , there were many other businesses situated throughout the town which were operated by 
Chinese residents, including many cafes and laundries. The need for so many laundries was generated from the extensive use by restaurants of heavy linens, which required frequent cleaning. The Quon Sang Lung Laundry building was a rare and unique example of a wood frame, boomtown-fronted laundry building, typical of structures built by small-scale Chinese entrepreneurs. The structure's siting and orientation, with its boomtown front facing an alley, revealed the history of discrimination faced by Chinese merchants in Fort Macleod. Through its location at the edge of the Provincial Historic Area, and segregation from the historic downtown with a rear-facing entrance, the Quon Sang Lung Laundry contributes to the historic narrative of immigrant populations in Fort Macleod, and the overall significance of the Provincial Historic Area.

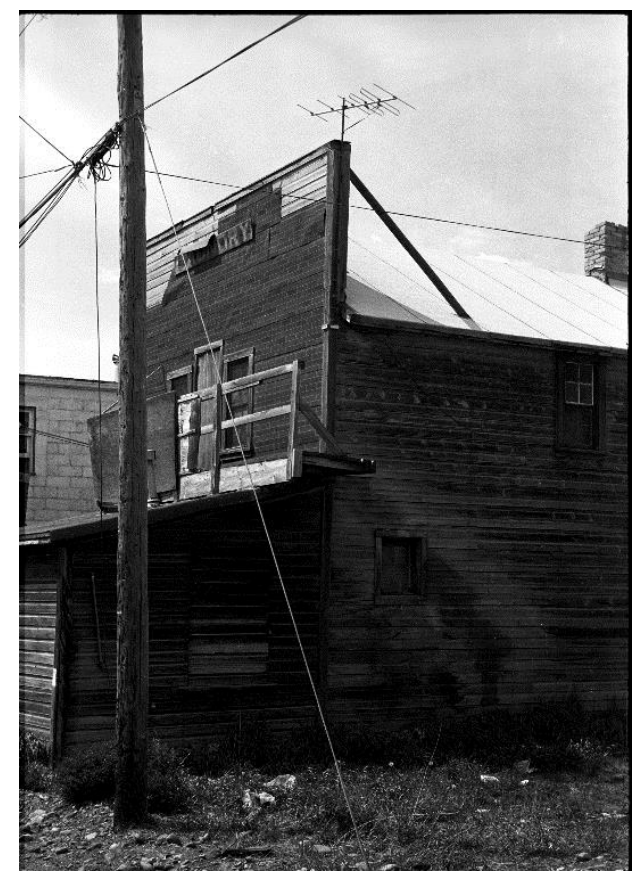

Figure 1: Partial south (front) and east facades, with brick chimney, Insulbrick cladding, shed addition \& wood balcony, 1973. Source: HRM

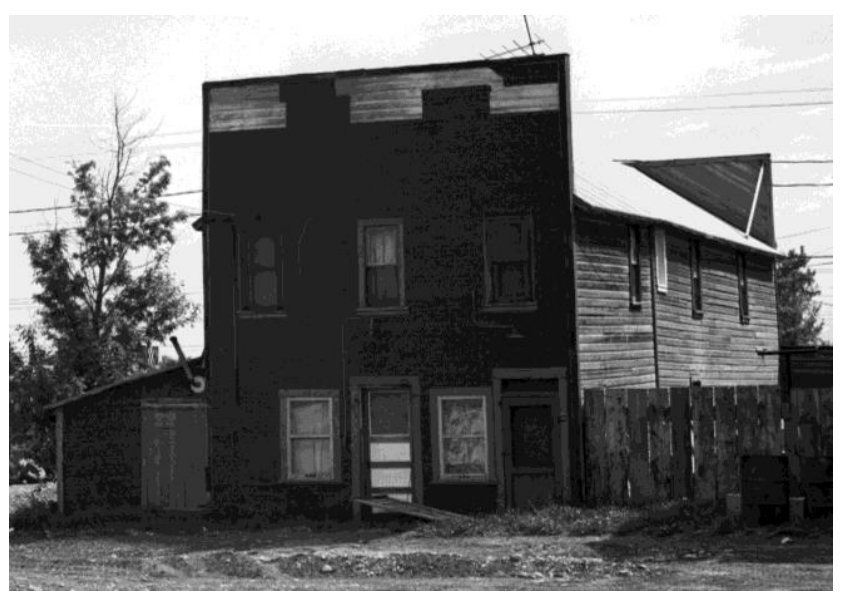

Figure 2: Addition at rear façade with boomtown storefront addressing 25 Street; shed at east side; Insulbrick cladding, regular fenestration pattern, (2) entrances, 1973. Source: HRM

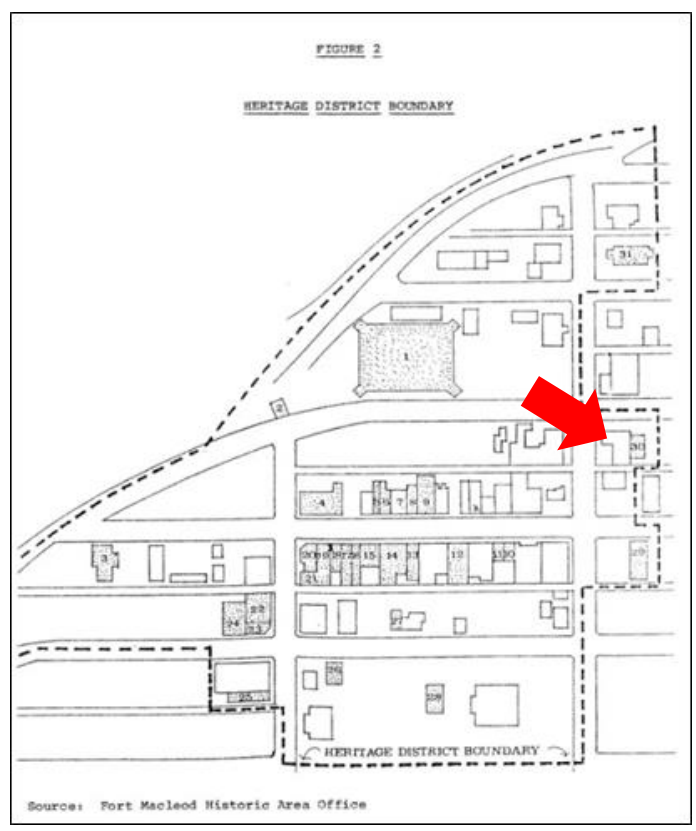

Figure 3: Fort Macleod Provincial Historic Area boundary map. Source: HRM

The Chinese laundry building was home to a diversity of businesses following its construction. In addition to being a laundry, it also housed a store selling herbs. During the 1930s, the building remained vacant. In 1945, it served as a garage for a short period then it was used to operate as a laundry and boarding house for a subsequent period with distinct ownerships until 1968. The building was used afterwards as a storage area until its demolition in January of 2017.

The workmanship on the exterior was demonstrated in the construction of the simple yet resilient dimension lumber structure. The interior revealed the installation of plain, functional wall finishes; undecorated interior window casings; and original wood strip flooring. The pattern and variety of early floor finishes, wall finishes and paint colours at the second floor was clearly an indication of its previous occupation as a boarding house. The building was a contributing element to the Fort Macleod Provincial Historic Area, which itself is significant for preserving Fort Macleod's vibrant pre-1914 business and service core. The Chinese Laundry building was the last remaining structure of similar designs in Chinatown, all of which were used as boarding houses or laundries by the Chinese community. 


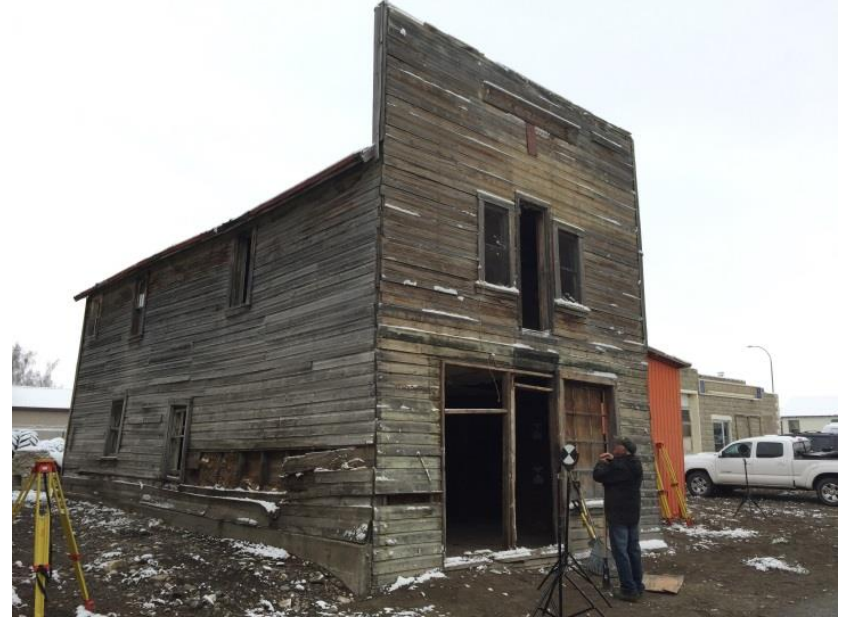

Figure 4: South and west facades. November, 2016.

\section{DIGITAL DOCUMENTATION}

In recent years, a condition assessment of the structure indicated that the building was not safe, and that the extent of the instability could lead to a sudden collapse. A demolition permit was issued by the Town and was scheduled for late 2016. Due to the imminent demolition of the building, there was only a short time frame to develop a documentation strategy. The recording of all the information of the remains of the original structure was crucial to preserve the historical memory of the building. On December 2016 Alberta Culture and Tourism (ACT) engaged the Departments of Anthropology and Archaeology and Geomatics Engineering from the University of Calgary, to digitally preserve the laundry building. The partnership with the university was vital, in order to be able to conduct the documentation in a timely manner. This project also provided graduate students in Archaeology and Geomatics Engineering with a unique opportunity to exercise their skills in a real world case-study.This project forms the basis of a collaborative arrangement developed between the University of Calgary and Alberta Culture and Tourism. The goal is to establish a regulatory trigger, so that the University of Calgary research group will be called whenever a heritage resource is at imminent risk, and in need of digital documentation.

Reality capture technologies such as terrestrial laser scanning are currently being used to digitally preserve heritage sites throughout the world - especially those at risk (Dawson et al., 2013, Dawson et al., 2009; Cesaro et al., 2012). Terrestrial laser scanners measure the amount of time taken for pulses of laser light to leave the scanner, strike the surface of an object within its range $(130 \mathrm{~m})$, and make its return. The scanner then calculates the pulse's horizontal and vertical angles, giving accurate $\mathrm{x}, \mathrm{y}$ and $\mathrm{z}$ coordinates. This is done millions of times, creating a dense three-dimensional cloud of points in the instrument's coordinate system that effectively captures objects within its field of view, such as buildings, artifacts, and landscape features. The resulting data can be archived online and used to create 2D architectural plans for documentation, restoration and even rebuilds. Photorealistic 3D models can also be created from laser scanning data and used to develop online virtual exhibits for communicating the historic and cultural significance of buildings and other features lost to destructive agents.

At the time of its documentation, the Quan Sang Lung Laundry building was located behind a local tire shop, where it was used as storage. At the request of ACT, the owner removed all of the tires, rims, and other materials stored within the building.
Heritage conservation technologists from ACT then visited the site, along with the research team from the University of Calgary, to develop a methodology for recording the building.

\subsection{Methodology}

Drone-based photogrammetry was used to capture the building in its current urban context, capturting its location relative to other nearby buildings. A DJI Phantom 4 drone was flown in an overlapping grid pattern over the area containing the laundry shop. The resulting images were processed to create a point cloud, mesh and final 3D model (https://skfb.ly/VWnE). Photogrammetry is an excellent way of creating visual models of artifacts and buildings. However, the accuracy and precision of these models is often too coarse to allow for the calculation of volumetric and linear measurements. Terrestrial laser scanners can provide point clouds with millimeter and submillimeter accuracy.

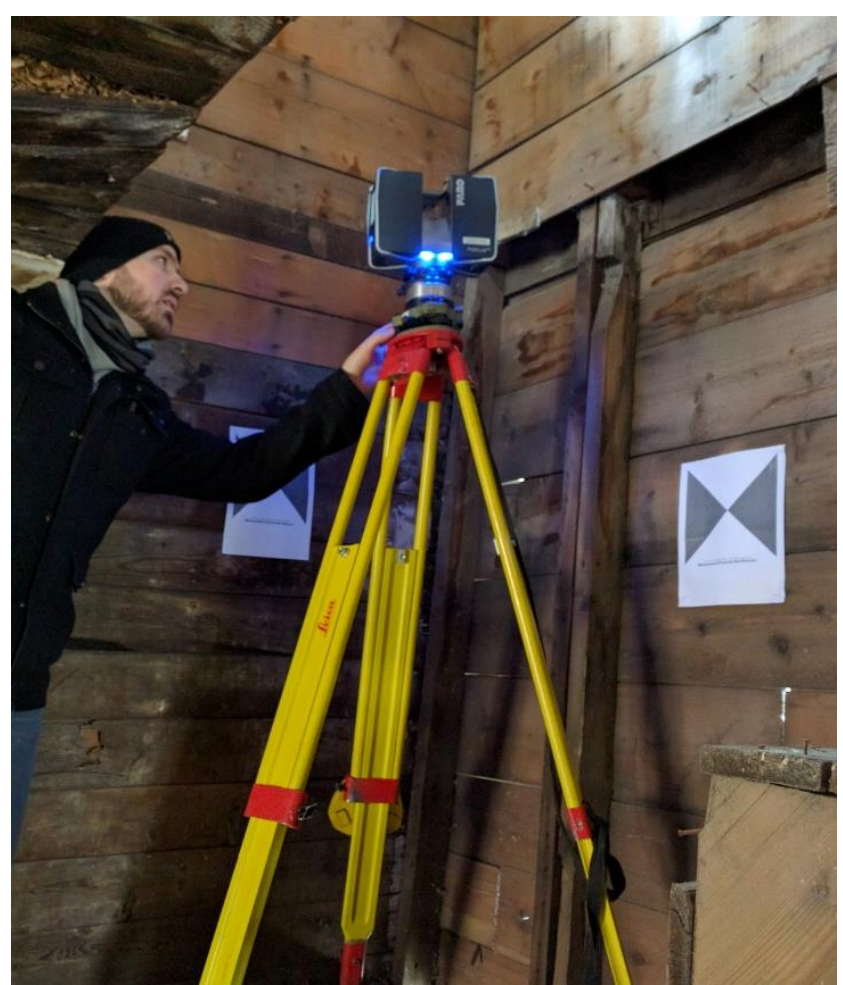

Figure 5: Scanning the interior of the laundry shop using the Faro Focus 3D scanner. Source: Peter Dawson.

Two laser scanners were used to record the building $-\mathrm{a} Z+\mathrm{F}$ 5010X scanner, and a Faro Focus 3D scanner (Figure 5). A series of paddle and spherical targets were placed at various locations around the structure, and well as within the main and top floors of the building. To ensure accurate point cloud registration, a minimum of three targets need to be common to two or more overlapping scans. Targets are not always necessary with the auto-registration algorithms found in programs such as Leica Cyclone, but they increase accuracy. Since one of the goals of the project was to create a set of architectural plans, the use of targets for registering the data was and essential part of the work flow. Figure 6 shows the fully registered point cloud for the building. 


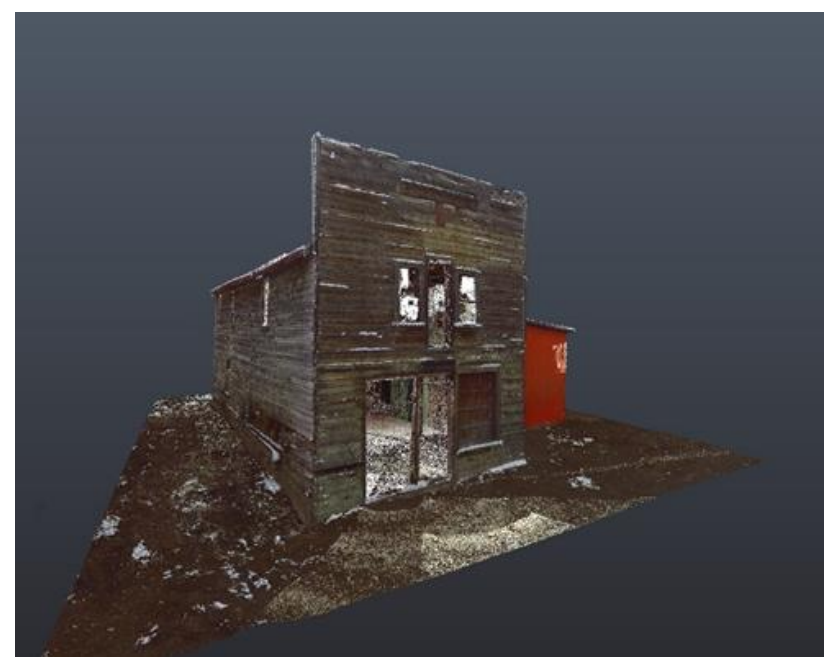

Figure 6: The registered point cloud for the complete building. Source: Peter Dawson

Recording of the structure was done over a period of 4.5 hours, during light snow, and a temperature that hovered around 0 degrees Celsius. Detailed recording the structure using hand measuring tapes, as is the common practice for recording historic structures in Alberta, would have taken much longer. Furthermore, the resulting data would have likely been prone to human errors in measurements

One of the advantages of the $\mathrm{Z}+\mathrm{F} 5010 \mathrm{X}$ scanner is that it allows the operator to register the point cloud data in real time, as the scanning is performed. This is advantageous because it allows the operator to identify if there are any gaps in the data in other words, the operator knows immediately if a critical area of the building has missed by the scanner. It also speeds postprocessing of the scanning data, once the operator returns to the office. As the owner was anxious to begin demolition of the structure as soon as possible, we were able to quickly inform ACT that they could give the "go-ahead" because the quality of the data was excellent, and the building had been fully captured.

Many heritage agencies have guidelines for recording historic structures. However, no such guidelines presently exist for using terrestrial laser scanners in the Province of Alberta, as well as many other regions of Canada. Therefore, one of the outcomes of this project was to begin to develop a workflow and a set of best practices for recording heritage structures using this technology.

\subsection{Creating 3D Models Architectural Plans}

In order to produce detailed architectural plans, a 3D model was first created by importing the point clouds into Rhino $5-$ a popular 3D modeling software package. The point clouds were used as a reference for extant modeling of the building in 3D. The point clouds were used in tandem with high resolution photos taken of the building during scanning. Interior details such as stairs and columns were added, and the 3D modeled roof was then paired with the rest of the building. A layer of textures and other details were next added to the model - the photographs taken onsite were especially helpful in this regard. Damaged areas of the building, such as parts of the exterior, floor, window frames, etc., were modeled separately and applied to the main model of the building. These additional layers were then textured and materialized as well. The final model was rendered in Rhino 5 using Vray to produce the images of the building. These images were further processed in
Photoshop C5 so that they looked as close as possible to the actual building (Figures 7,8).

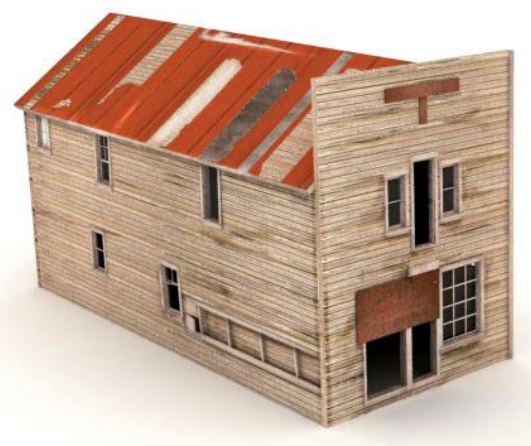

Figure 7: The completed 3D model of the laundry shop. Source: Peter Dawson

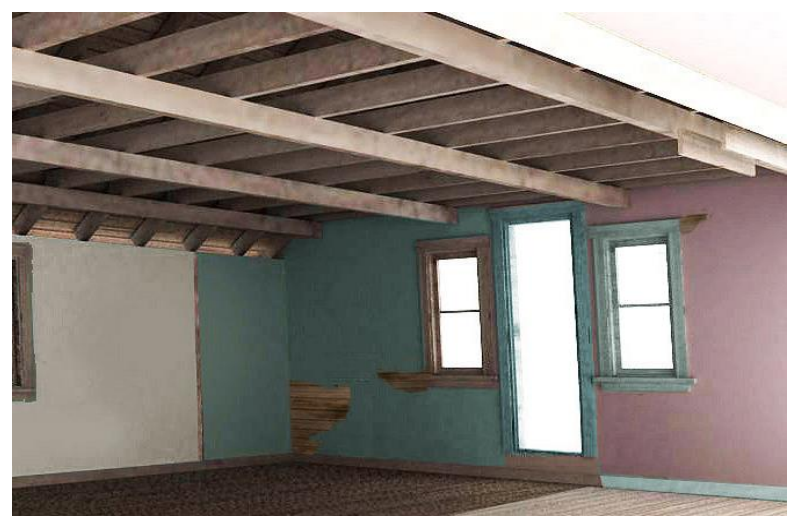

Figure 8: Photorealistic reconstruction of the second floor of the laundry shop. Source: Peter Dawson

The model was then exported from Rhino 5 into AutoCAD 2016 to produce the construct 2D drawings of the building. The tilted structure and crooked walls were corrected in consultation with the heritage managers at Alberta Culture and Tourism. The drawings were then layered. The drawing lines were given weight based on the guidelines provided by Alberta Culture and Tourism (Figure 9). This process helped to retain both the extant and construct versions of drawings from basically a single model and a single platform, i.e., Rhino 5 . Only the postproduction work required using Photoshop and AutoCAD.

\subsection{Creating a Digital Heritage Archive.}

A website was created to archive the data and make it accessible to heritage concervation technologiests at ACT. This includes delivery of accurate 3D renders of the exterior and interior spaces and a collection of architectural drawings comprising floor plans, sections, and elevations. A Virtual Reality App was also developed for use with Google cardboard. The VR app allows users to teleport to various locations around the exterior and interior of the 3D model, and can be downloaded directly from the website/archive. The resulting documents and point cloud data collected during the fieldwork will be archived by the Government of Alberta and will be used for educational purposes as well as to support future initiatives in Fort Macleod.

\section{CONCLUSIONS}


This project represents our first steps in developing a preliminary set of guidelines for the collection, registration, and archiving of laser scanning data and three dimensional models for heritage sites at risk in the Province of Alberta. A set of guidelines will be produced with references to Historic England's Metric Survey Specifications for Cultural Heritage, but will be modified based on local experience, especially the digital preservation project of the Chinese laundry. The revised guidelines will better suit the nature of Alberta's unique heritage, as well as the needs of Alberta Culture and Tourism.

The workflow we have developed also allows for a rapid and thorough documentation of a heritage building and site in a relatively short period of time. The scanning of the building was done over a period of 4 hours. It would have taken a great deal longer for the same data to have been recorded by hand. Furthermore, such recording would have been prone to human error.
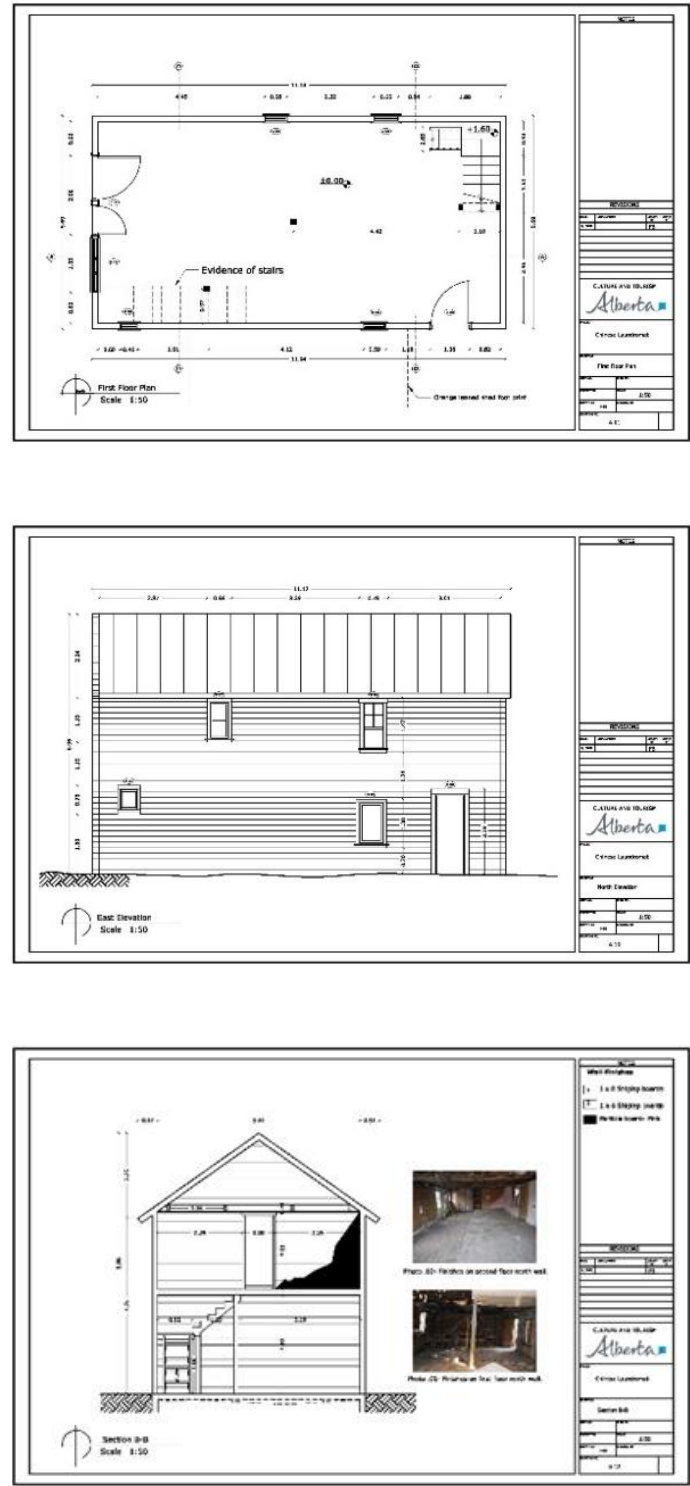

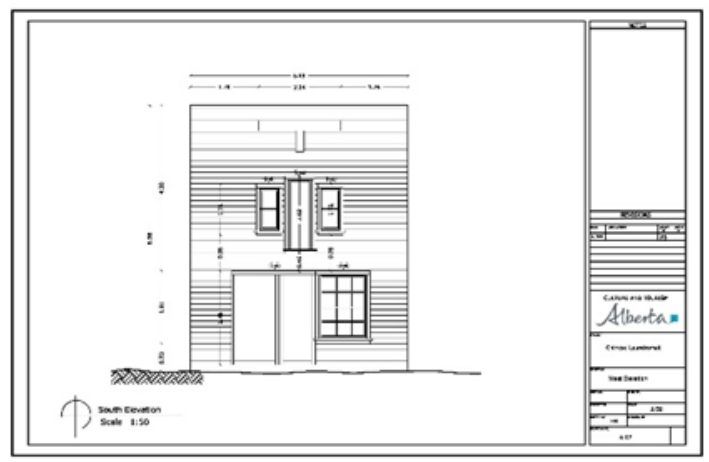

Figure 9: Examples of architectural plans created by Farzan Baradaran from the point cloud data. Source: Peter Dawson

As Figure 10 vividly illustrates, the Quan Sang Lung Laundry building no longer stands in Fort Macleod. However, through digital modeling, the work done by researchers and students from $\mathrm{U}$ of $\mathrm{C}$ and ACT guarantees that a three-dimensional representation of the building is available for future use. To this end, we have set up a website contaning architectural plans, 3D models, and a downloadable VR app for Google Cardboard (www.oklaundry.org). The Quon Sang Lung Laundry shop exists as monument to the enduring hardships suffered by Chinese laborers, who were excluded by government policies, yet worked hard to establish themselves and their communities in towns and cities across Canada. It is therefore essential that buildings asscoiated with the immigrant experience be preserved - if not physically, then virtually, so that their legacies and histories can be accessed by future generations.

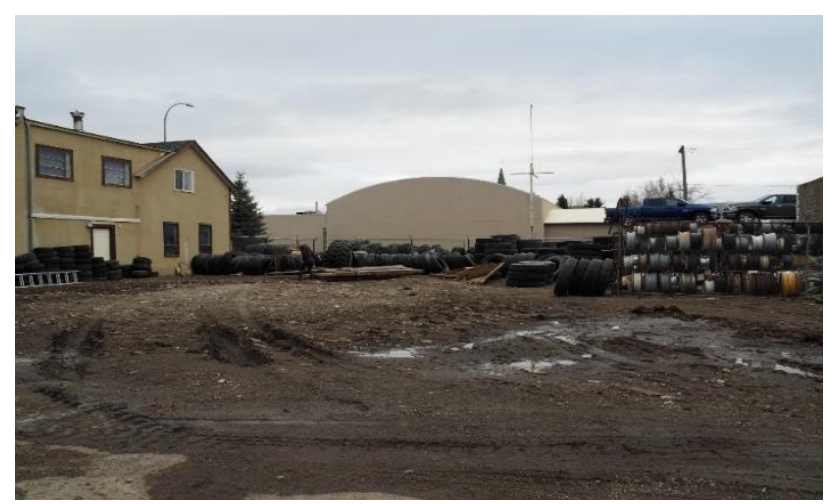

Figure 10: The Quon Sang Lung Laundry building site following demolition of the structure. Source: Peter Dawson

\section{REFERENCES}

Cesaro, G., Fakhoury, L., Paolini, A., Santana Quintero, M., Vadafari, A., Van Balen, K., Vileikis, O., 2012. Risk Management at Heritage Sites: a case study of the Petra World Heritage Site. United Nations Educational, Scientific and Cultural Organization (UNESCO), Paris, France; Katholieke Universiteit, Leuven \& UNESCO Amman Office.

Dawson, P., Levy, R.M., Oetelaar, G., Arnold, C., Lacroix, D., Mackay, G., 2009. Documenting Mackenzie Inuit Architecture Using 3D Laser Scanning. Alaska Journal of Anthropology, Vol. 7, Issue 2. Pp. 22-44. 
Dawson, P., Bertulli, M., Levy, R., Tucker, C., Dick, L.,

Cousins, L.P., 2013 Application of 3D Laser Scanning to the Preservation of Fort Conger, a Historic Polar Research Base on Northern Ellesmere Island, Arctic Canada. Arctic Vol. 66, Issue 2. Pp. 147-158.

Marshall, A.R., 2009. Manitoba History: Early Chinese Settlers in Western Manitoba. Manitoba Historical Society, Number 62, Winter 2009.

Morrison, J.H., Bell, G., and Lee, A., 2013. Chinese Migration to the Maritimes: The Early Years, 1890-1947. Journal of the Royal Nova Scotia Historical Society, Vol. 16.

Quon Sang Lung Laundry, Statement of Significance. Alberta Culture and Tourism, Historic Resources Management Branch (File: Des. 2334).

Hoe, Ban Seng., 2003. Enduring Hardship: The Chinese Laundry in Canada. Canadian Museum of Civilization, Ottawa.

Wang, J., 2002. Gender, Race and Civilization: The Competition between American Power Laundries and Chinese Steam Laundries, 1870's-1920's. American Studies International, Vol. 40, No. 1. Pp. 52-73. 